\title{
Synthesis of Some Novel Derivatives of 2-(9H-purin-6-ylsulfanyl) Acetohydrazide as Potential Antithyroid Agents
}

\author{
Ismat Fatima, ${ }^{2}$ Munawar A. Munawar, Waqar Nasir, Misbahul A. Khan, ${ }^{1}$ Affia Tasneem, ${ }^{2}$ Tauqir Fatima ${ }^{3}$ \\ and Muhammad R. Adil ${ }^{4}$ \\ 1 Institute of Chemistry, University of the Punjab, Lahore, Pakistan. munawaralimunawar@yahoo.com \\ 2 CENUM, Mayo Hospital, Lahore, Pakistan \\ 3 Government College University, Lahore, Pakistan \\ 4 A.E.M.C., Lahore, Pakistan
}

Received December 29, 2011; Accepted May 25, 2012

\begin{abstract}
Some novel derivatives of 2-(9H-Purin-6-ylsulfanyl)acetohydrazide were synthesized by reacting it with respective aldehydes in ethanol. The antithyroid effect of these compounds was ascertained in vitro by studying their complexation with iodine spectrophotometrically. In vivo, the hormonal as well as histological variations in male Wistar rats demonstrated significant antithyroid potential $(p \leq 0.05)$ of these compounds.

Key words: Hyperthyroidism, Antithyroid activity, Hydrazides, In vitro activity, In vivo Activity.
\end{abstract}

\section{Introduction}

Hyperthyroidism is a condition characterized by over production of the thyroid hormones. It directly or indirectly disturbs the whole body including mental and physical growth [1]. The available treatments for hyperactive thyroid are thyroidechtomy (surgical removal of a part or whole gland), radioactive iodine therapy or use of antithyroid drugs. The former two modes though provide a permanent treatment but have serious set backs like perpetual hypothyroidism as well as complications arising out of surgery like tracheal suppressing etc. Moreover, there are certain hyperthyroid conditions which are not permanent and do not need surgery or radio iodine therapy; the use of antithyroid drugs becomes the only choice in such conditions [2]. On the other hand, there is a scarcity of drugs in this particular area. The widely used synthetic antithyroid agents (SATs) like propylthiouracil, methimazole and carbimazole belong to sulfonamide group and have serious side effects [3]. Keeping in view the scarcity and side effects of the existing drugs, many compounds were evaluated and some were reported to possess recognizable antithyroid activity [4]. It has been observed that compounds capable of making stable charge transfer complex with iodine and having formation constant $\left(\mathrm{K}_{\mathrm{c}}\right)$ values $\geq 100 \mathrm{~L} \mathrm{~mol}^{-1}$ generally exhibit antithyroid effects in vivo [5]. Keeping in view the ability of heterocyclic compounds, especially purines, to complex with iodine [6], certain purine derivatives were evaluated for potential antithyroid effects and significant antithyroid activity was observed in vivo [7]. 6-Mercaptopurine (9H-purine-6-thiol) and its alkyl derivatives were also found to inhibit thyroid activity via complexation with iodine [8]. 6-(Benzylsulfanyl)-9H-Purin
Resumen. Algunos nuevos derivados de la 2-(9H-purin-6-ilsulfanil)acetohidrazida fueron sintetizados mediante la reacción con los respectivos aldehídos en etanol. El efecto antitiroideo de estos compuestos se determinó in vitro mediante el estudio espectrofotométrico de sus complejos con yodo. In vivo, las variaciones hormonales e histológicas en ratas macho Wistar demostraron de manera significativa $(p \leq 0,05)$ el potencial antitiroideo de estos compuestos.

Palabras clave: Hipertiroidismo, actividad antitiroidea, hidracidas, actividad in vitro, actividad in vivo.

was found most active amongst the series, probably because of the presence of conjugation. So, we planned to synthesize a series of compounds with aromatic substituents to get enhanced activity. At the same time, biological activity of hydrazides and acetohydrazides has also been reported [9]. Therefore, it was planned to synthesize $N^{\prime}$-[substituted alkylphenylmethylidene]-2-(9H-purin-6-ylsulfanyl)acetohydrazides (3-11) as prospective SATs with 6-mercaptopurine as a starting material. The compounds (3-11) are expected to be novel as they are not found to be reported in the literature elsewhere. The Sulfanyl group, which is thought to be a cause of toxic side effects of many synthetic drugs, has been blocked in these compounds [10]. Therefore, it can be fairly assumed that these compounds would be less toxic. The antithyroid activity of purified compounds was evaluated in vitro by studying their complexation with iodine spectrophotometrically and in vivo in male Wistar rats.

\section{Results and Discussion}

\section{Synthesis}

Ethyl (9H-purin-6-ylsulfanyl)acetate (1) and 2-(9H-Purin-6ylsulfanyl)acetohydrazide (2) were synthesized by using both conventional and microwave assisted reactions. The use of microwave oven not only substantially reduced the reaction time but also resulted in higher yields and purer products. $N^{\prime}-$ [substituted phenylmethylidene]-2-(9H-purin-6-ylsulfanyl)acet ohydrazide (3-11) were synthesized by the reaction of 2 with substituted aryl aldehydes in ethanol (Scheme 1). 

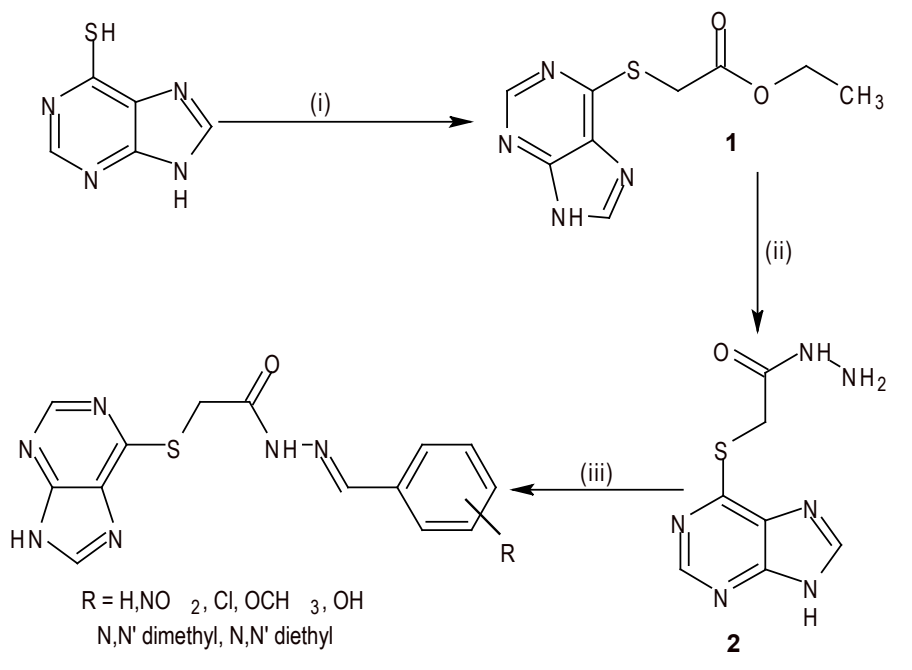

3- 11

Scheme 1. Reagents and conditions: (i) $\mathrm{Et}_{3} \mathrm{~N}, \mathrm{ClCH}_{2} \mathrm{COOCH}_{2} \mathrm{CH}_{3}$, EtOH, 6 hour reflux; (ii) $\mathrm{NH}_{2} \mathrm{NH}_{2} \cdot \mathrm{H}_{2} \mathrm{O}, \mathrm{EtOH}, 5$ hour reflux. (iii) aryl aldehyde, EtOH, reflux 4-6 hours.

\section{Assessment of antithyroid activity}

\section{In Vitro}

The solutions of pure compounds in DMSO have absorption maxima in UV region and spectral data indicate the complex formation mostly at $265-70 \mathrm{~nm}$ (Table 1). All the compounds except 2, demonstrated 1:1 charge transfer (CT) complexation with iodine. However, 2 also exhibited complex formation near $315 \mathrm{~nm}$. The compounds exhibited $\mathrm{K}_{\mathrm{c}}$ values well above $100 \mathrm{~L}$ $\mathrm{mol}^{-1}$, which indicate their good antithyroid potential.

It is observed that the stability of the complex with iodine not only depends upon the substituents but also on their location in the ring like the presence of a nitro group at meta position (4) resulted in more stable complex as compared to ortho (5) and para (3) positions.

In vivo

In vivo the blood assays showed decrease of varying degree in $\mathrm{FT}_{3}$ and $\mathrm{FT}_{4}$ levels with a corresponding increase in Thyroid-stimulating hormone (TSH) concentrations for the animals treated with the compounds (1-11) as compared to control and vehicle control (Table 2$)$. The hormonal variations were quite significant $(p \leq 0.05)$.

The antithyroid potential of 3-11 was found to be weaker than methimazole, which was introduced as "positive control" in the study yet some compounds like 3, $\mathbf{4}$ and $\mathbf{8}$ exhibited comparable antithyroid effects.

The Line-Column Plot between the percentage hormonal variations versus $\mathrm{K}_{\mathrm{c}}$ values revealed a positive correlation between the formation constants and antithyroid activity in vivo (Fig. 1).

The histological studies also revealed the antithyroid effects of the compounds as the follicular epithelia in the treated animals were found to be cylindrical with larger nuclei rather than to be of cuboidal shape like in control animals (Fig. 2).
Table 1. Spectrophotometric data of CT complexes between compounds and $\mathrm{I}_{2}$.

\begin{tabular}{cccc}
\hline Compound No. & ${ }^{*} \mathrm{CTB} / \mathrm{nm}$ & ${ }^{\dagger} \mathrm{K}_{\mathrm{c}} / \mathrm{L} \mathrm{mol}^{-1}$ & ${ }^{\dagger} \varepsilon_{\mathrm{c}} / \mathrm{L} \mathrm{mol}^{-1} \mathrm{~cm}^{-1}$ \\
\hline $\mathbf{1}$ & 270 & $0.390 \times 10^{4}$ & $1.144 \times 10^{5}$ \\
$\mathbf{2}$ & 315 & - & - \\
$\mathbf{3}$ & 265 & $0.706 \times 10^{4}$ & $0.569 \times 10^{5}$ \\
$\mathbf{4}$ & 265 & $1.989 \times 10^{4}$ & $0.497 \times 10^{4}$ \\
$\mathbf{5}$ & 275 & $0.542 \times 10^{4}$ & $3.474 \times 10^{4}$ \\
$\mathbf{6}$ & 310 & $0.671 \times 10^{4}$ & $5.769 \times 10^{4}$ \\
$\mathbf{7}$ & 305 & $0.443 \times 10^{4}$ & $7.032 \times 10^{4}$ \\
$\mathbf{8}$ & 270 & $1.304 \times 10^{4}$ & $4.504 \times 10^{4}$ \\
$\mathbf{9}$ & 270 & $0.685 \times 10^{4}$ & $3.454 \times 10^{4}$ \\
$\mathbf{1 0}$ & 310 & $0.970 \times 10^{4}$ & $6.134 \times 10^{4}$ \\
$\mathbf{1 1}$ & 320 & $0.989 \times 10^{4}$ & $0.3504 \times 10^{4}$ \\
\hline
\end{tabular}

* Charge transfer band.

${ }^{\dagger}$ Formation constant.

$\$$ Molar extinction coefficient.

Table 2. The mean hormonal concentrations of different animal groups after 15 days.

\begin{tabular}{ccccccc}
\hline \multirow{2}{*}{ Animal Groups } & \multicolumn{2}{c}{$\mathrm{FT}_{3}$} & \multicolumn{2}{c}{$\mathrm{FT}_{4}$} & \multicolumn{2}{c}{$\mathrm{TSH}$} \\
\cline { 2 - 7 } & \multicolumn{2}{c}{$\mathrm{pmol} / \mathrm{L}$} & \multicolumn{2}{c}{$\mathrm{pmol} / \mathrm{L}$} & \multicolumn{2}{c}{$\mu \mathrm{i} . \mathrm{u} . / \mathrm{mL}$} \\
\cline { 2 - 7 } & Mean & S.D. & Mean & S.D. & Mean & S.D. \\
\hline Control & 9.68 & 1.2 & 38.42 & 2.39 & 1.88 & 0.26 \\
Vehicle Control & 9.07 & 1.29 & 37.19 & 2.80 & 1.96 & 0.23 \\
$\mathbf{1}$ & 7.83 & 0.97 & 32.46 & 4.49 & 3 & 0.89 \\
$\mathbf{2}$ & 6.72 & 1.40 & 31.66 & 3.38 & 2.9 & 0.32 \\
$\mathbf{3}$ & 7.02 & 1.41 & 32.69 & 3.29 & 3.35 & 0.96 \\
$\mathbf{4}$ & 5.72 & 2.04 & 25.3 & 3.54 & 3.73 & 0.73 \\
$\mathbf{5}$ & 7.24 & 1.25 & 32.76 & 4.07 & 2.86 & 0.79 \\
$\mathbf{6}$ & 7.12 & 0.96 & 31.28 & 3.54 & 2.98 & 0.56 \\
$\mathbf{7}$ & 7.47 & 1.64 & 31.1 & 4.35 & 2.95 & 0.49 \\
$\mathbf{8}$ & 6.27 & 0.96 & 27.88 & 3.75 & 3.26 & 0.93 \\
$\mathbf{9}$ & 7.18 & 0.85 & 32.16 & 4.19 & 2.96 & 0.47 \\
$\mathbf{1 0}$ & 6.72 & 1.33 & 30.33 & 3.59 & 3.05 & 0.64 \\
$\mathbf{1 1}$ & 6.7 & 1.77 & 28.66 & 5.74 & 3.08 & 0.80 \\
Methimazole & 5.12 & 1.10 & 24.86 & 5.97 & 4.26 & 1.18 \\
\hline
\end{tabular}

Dose rate $=20 \mathrm{mg} / \mathrm{kg} /$ day via i.p. injection, $\mathrm{n}=5$.

Treatment duration $=15$ days; Assays in duplicate.

Moderate to severe colloid depletion and follicular hyperplasia and hypertrophy were also observed in the treated animals as compared to the control groups.

Thyroid body index (TBI) is a useful parameter defined as weight of clean thyroid tissues (mg) per $100 \mathrm{~g}$ of final body weight of the animal. These indices for the treated animals were found quite higher than the controls, which show the increased glandular activity. In fact, the compounds created artificial iodine deficiency and retarded the biosynthesis of thyroid hor- 


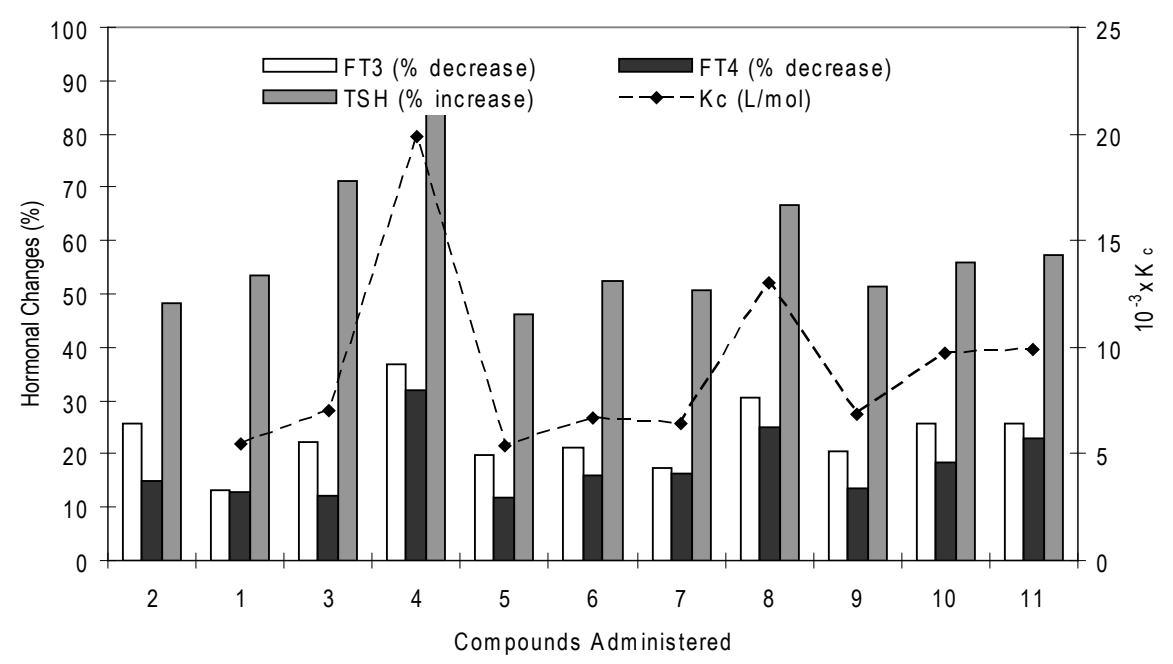

Fig. 1. Positive linear relation between percentage hormonal variations and the values of the formation constants.
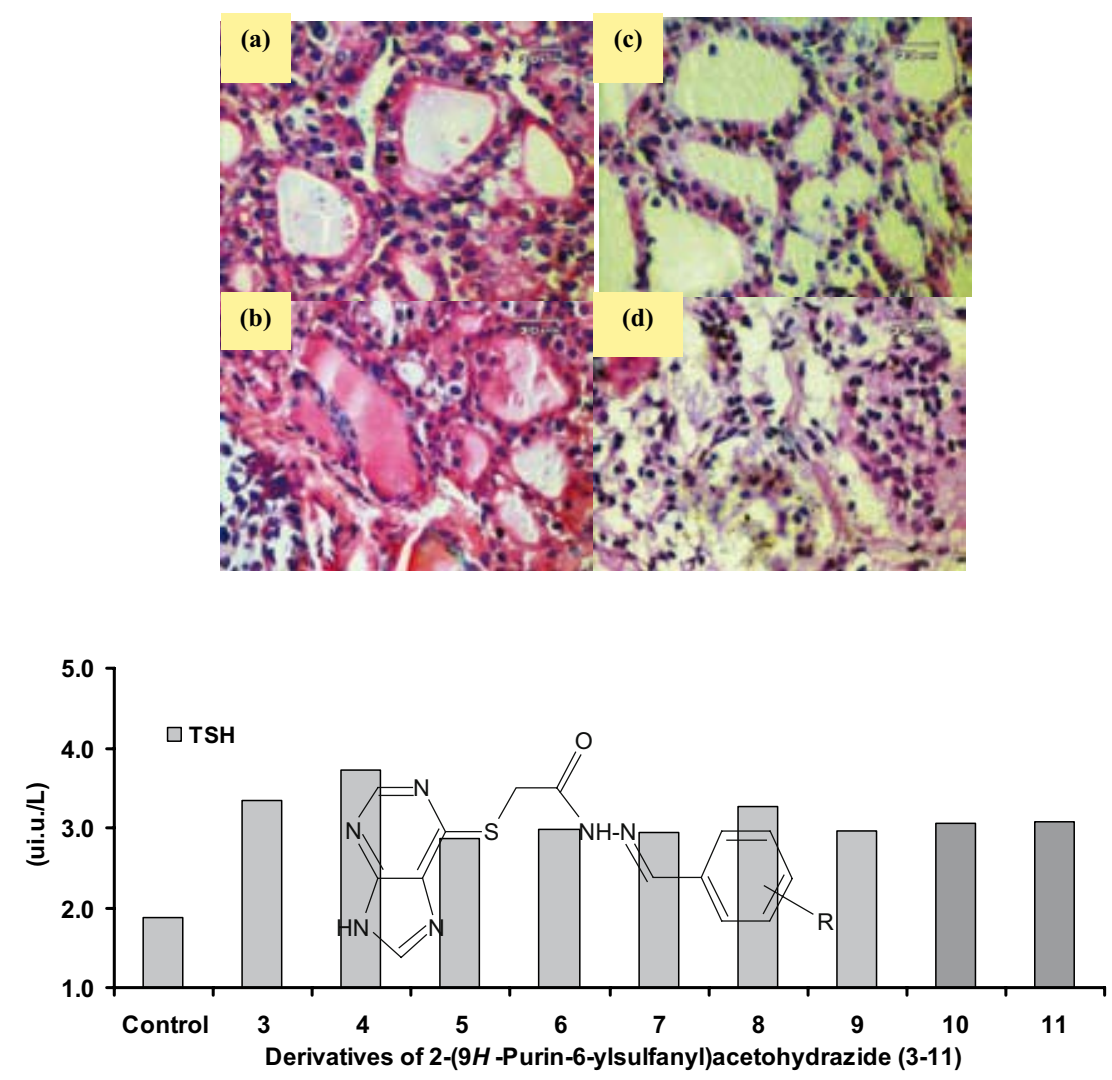

Fig. 2. Thyroid sections of (a) control, (b) 1, (c) 3, (d) 4 clearly depicting thyroid hyperplasia and cylindrical epithelium.

mones. In response to the decreased hormonal concentrations in the blood, the pituitary released more quantities of TSH thus directing the gland to produce more hormones. The gland responded accordingly by increasing the number (hypertrophy) and size of the follicular cells (hyperplasia) and consuming the colloid storage to produce more hormones. The cylindrical shape as compared to the cuboidal allows more cells per unit volume of the tissue, which in turn increases the thyroid weight (TBI). The follicular cell hyperplasia, hypertrophy, TBIs and colloid depletion as observed under microscope are tabulated (Table 3) which indicate fair to high antithyroid effects of the compounds. 
Table 3. Histological observations of thyroid from different groups of animals after 15 days study period.

\begin{tabular}{|c|c|c|c|}
\hline $\begin{array}{l}\text { Animal Group/ } \\
\text { Description }\end{array}$ & $\begin{array}{c}\text { Colloid } \\
\text { Depletion }\end{array}$ & $\begin{array}{c}\text { TBI } \\
(\mathrm{mg} / 100 \mathrm{~g})\end{array}$ & $\begin{array}{c}\text { Follicular Cell } \\
\text { Hypertrophy/Hyperplasia }\end{array}$ \\
\hline Control & Nil & 5.41 & Nil \\
\hline Vehicle Control & \pm & 6.07 & + \\
\hline 1 & + & 9.43 & ++ \\
\hline 2 & ++ & 9.61 & ++ \\
\hline 3 & + & 10.37 & ++ \\
\hline 4 & +++ & 12.63 & +++ \\
\hline 5 & ++ & 8.41 & ++ \\
\hline ర્ષ્త & + & 9.07 & ++ \\
\hline$\stackrel{\varrho}{\varrho}$ & + & 9.15 & ++ \\
\hline 8 & ++ & 11.45 & +++ \\
\hline 9 & ++ & 8.56 & ++ \\
\hline 10 & +++ & 10.33 & ++ \\
\hline 11 & +++ & 10.54 & ++ \\
\hline MMI & +++ & 14.91 & +++ \\
\hline
\end{tabular}

\pm Slight; + Mild; ++ Moderate; +++ Severe.

\section{Conclusion}

Derivatives of 2-(9H-purin-6-ylsulfanyl)acetohydrazide (3-11) possess notable antithyroid effects with some being more active than others. Very stable charge transfer complexation with iodine and significant hormonal variations in vivo suggests further study on these compounds from cytotoxic point of view, which may lead to the discovery of a new drug.

\section{Experimental section}

\section{Synthesis}

Reagent grade materials were purchased from Aldrich Chemical Co. and were used without further purification. Melting points were determined using a Gallenkamp melting point apparatus and are uncorrected. ${ }^{1} \mathrm{H}$ NMR spectra were taken in DMSO- $d_{6}$ and recorded at 300, 400 and $500 \mathrm{MHz} .{ }^{13} \mathrm{C}$ NMR spectra were taken in 75 and $100 \mathrm{MHz}$ on a Brucker/XWIN-NMR instrument. Chemical shifts are given in ppm relative to $(\mathrm{Me})_{4} \mathrm{Si}$ as internal standard (abbreviations: s, singlet; $d$, doublet; $t$, triplet; $\mathrm{m}$, multiplet). Mass spectra were recorded on JEOL MS Route. Elemental analyses were carried out on Perkin Elmer 2400CHN Analyzer performed in H.E.J. Research Institute, Karachi University, Karachi and were within the range of $\pm 0.4 \%$ of predicted values for all the compounds. UV/VIS Spectra were recorded on a double beam UVD-3500 spectrophotometer (Labomed Inc.). Modified House hold microwave system (900 watts) was used for microwave assisted synthesis. Thin Layer chromatography was carried out on Merck silica gel $60 \mathrm{~F}_{254}$ plates. Iodine was obtained from Merck (suprapur) bisublimed and was kept in dark in a dessicator containing $\mathrm{P}_{2} \mathrm{O}_{5}$. DMSO of spectroscopic grade was obtained from Merck, was dried over calcium hydride, distilled under reduced pressure and stored over type 4A 4 A molecular sieves. Radioimmunoassay (RIA) kits for free $\mathrm{T}_{3}$ and $\mathrm{T}_{4}$ were obtained from Immunotech (France) and TSH for rat ELISA kit from Cusabio Biotech Ltd. (China) and blood assay was carried out at Centre for Nuclear Medicine Lahore (Pakistan).

\section{Ethyl (9H-purin-6-ylsulfanyl)acetate (1)}

Conventional Method: 6-Sulfanylpurine monohydrate $(0.170 \mathrm{~g}$, $1 \mathrm{mmol})$, triethylamine $(0.14 \mathrm{~mL}, 1 \mathrm{mmol})$ and ethyl chloroacetate $(0.18 \mathrm{~mL}, 1.1 \mathrm{mmol})$ in ethanol $(10 \mathrm{~mL})$ was refluxed for six hours. Ethanol was removed under reduced pressure and the product was recrystallized from ethanol. Purity of all reported compounds is more than $95 \%$.

Microwave Assisted Synthesis. 6-Sulfanylpurine monohydrate $(0.170 \mathrm{~g}, 1 \mathrm{mmol})$, triethylamine $(0.14 \mathrm{~mL}, 1 \mathrm{mmol})$ and ethyl chloroacetate $(0.18 \mathrm{~mL}, 1.1 \mathrm{mmol})$ in ethanol $(10 \mathrm{~mL})$ were irradiated in modified household microwave oven $(900$ watts) equipped with water condenser for five minutes. The reaction was worked up as mentioned above.

Amorphous powder: $\mathrm{mp} 110^{\circ} \mathrm{C}$; Yield conventional $63 \%$; microwave 91\%; ${ }^{1} \mathrm{H}$ NMR $\delta\left(300 \mathrm{MHz}, \mathrm{DMSO}-\mathrm{d}_{6}\right) 13.51(1 \mathrm{H}$, s), $8.60(1 \mathrm{H}, \mathrm{s}), 8.47(1 \mathrm{H}, \mathrm{s}), 4.16(2 \mathrm{H}, \mathrm{qt}, \mathrm{J}=7.20 \mathrm{~Hz}), 3.90$ $(1 \mathrm{H}, \mathrm{s}), 1.20(3 \mathrm{H}, \mathrm{t}, \mathrm{J}=7.2 \mathrm{~Hz}) ;{ }^{13} \mathrm{C}$ NMR $(75 \mathrm{MHz}) \delta 169.8$, 154.6, 151.3, 151.2, 143.4, 131.4, 61.0, 30.7, 13.9; MS EI (m/z) $238\left(\mathrm{M}^{+}, 50 \%\right), 194$ (12), 193 (46), 192 (49), 167 (33), 165 (100); Anal: calculated for $\mathrm{C}_{9} \mathrm{H}_{10} \mathrm{~N}_{4} \mathrm{O}_{2} \mathrm{~S}$ (238.267) C, 44.99; $\mathrm{H}$, 5.03, N, 23.32\%, Found; C, 44.86; H, 4.99; N, 23.35\%.

\section{2-(9H-purin-6-ylsulfanyl)acetohydrazide (2)}

Conventional Method. Ethyl (9H-purin-6-ylsulfanyl)acetate $(0.256 \mathrm{~g}, 1 \mathrm{mmol})$ and hydrazine hydrate $(0.12 \mathrm{~mL}, 2.5 \mathrm{mmol})$ in ethanol $(10 \mathrm{~mL})$ were refluxed for five hours. Reaction was monitored by TLC. After the completion of reaction, solution was concentrated and refrigerated overnight. White precipitates obtained on cooling, were dried and recrystallized in ethanol.

Microwave Assisted Synthesis: Ethyl (9H-purin-6ylsulfanyl)acetate $(0.256 \mathrm{~g}, 1 \mathrm{mmol})$ and hydrazine hydrate $(0.12 \mathrm{~mL}, 2.5 \mathrm{mmol})$ were dissolved in ethanol $(10 \mathrm{~mL})$. The reaction flask was irradiated in microwave oven for three minutes. Reaction was monitored by TLC, white precipitates obtained were dried and recrystallized in ethanol.

White powder: $\mathrm{mp} 176{ }^{\circ} \mathrm{C}$ (dec.); Yield congenital 78\%; microwave 92\%; ${ }^{1} \mathrm{H}$ NMR $\delta\left(300 \mathrm{MHz}, \mathrm{DMSO}-\mathrm{d}_{6}\right) 9.35(1 \mathrm{H}$, s), $8.65(1 \mathrm{H}, \mathrm{s}), 7.09(1 \mathrm{H}, \mathrm{s}), 6.26(1 \mathrm{H}, \mathrm{s}), 5.98(1 \mathrm{H}, \mathrm{s}) ;{ }^{13} \mathrm{C}$ NMR $\delta(100 \mathrm{MHz}$, DMSO-d 6 ) 166.7, 156.9, 151.9, 150.4, 143.6, 129.0, 30.4; MS EI (m/z) $224\left(\mathrm{M}^{+}, 29 \%\right), 193$ (35), 182 (27), 177 (12), 165 (30), 160 (28), 150 (100); Anal: calculated for $\mathrm{C}_{7} \mathrm{H}_{8} \mathrm{~N}_{6} \mathrm{OS}$ (224.244) C, 37.16; H, 4.45; N, 37.14\%, Found; C, 37.09; H, 4.42; N, 37.12\%.

N'-[Substituted Phenylmethylidene]-2-(9H-purin-6-ylsulfanyl) acetohydrazide (3-11)

General Procedure: A mixture of 2-(8,9-dihydro-7H-purin-6ylsulfanyl)acetohydrazide (1 mmol) and substituted benzalde- 
hydes $(1.1 \mathrm{mmol})$ in ethanol $(20 \mathrm{~mL})$ with few drops of acetic acid were refluxed for 4-6 hours. The progress of the reaction was monitored by TLC. The product obtained as precipitate was filtered hot and recrystallized from ethanol.

\section{$N^{\prime}-[(E)-(4-N i t r o p h e n y l) M e t h y l i d e n e]-2-(9 H-p u r i n-6-$ ylsulfanyl)acetohydrazide (3)}

$N^{\prime}$-[(E)-(4-nitrophenyl)methylidene]-2-(9H-purin-6-ylsulfanyl acetohydrazide was prepared from 2-(9H-purin-6-ylsulfanyl) acetohydrazide (2) $(0.226 \mathrm{~g}, 1 \mathrm{mmol})$ and 4-nitrobenzaldehyde $(0.166 \mathrm{~g}, 1.1 \mathrm{mmol})$ in ethanol $(20 \mathrm{~mL})$ with a few drops of acetic acid. Immediate reaction took place and yellow precipitates of the product were obtained.

Yellow solid: mp $263{ }^{\circ} \mathrm{C}$, Yield 98\%; ${ }^{1} \mathrm{H}$ NMR $\delta(400$ MHz, DMSO-d $\left.{ }_{6}\right) 12.08(1 \mathrm{H}, \mathrm{s}), 11.91(1 \mathrm{H}, \mathrm{s}), 8.67(1 \mathrm{H}, \mathrm{s})$, $8.66(1 \mathrm{H}, \mathrm{s}), 8.47-8.13(4 \mathrm{H}, \mathrm{m}), 7.96(1 \mathrm{H}, \mathrm{s}), 4.69(2 \mathrm{H}, \mathrm{s})$; ${ }^{13} \mathrm{C}$ NMR $\delta$ (100 MHz, DMSO-d 6 ) 169.4, 164.4, 151.3, 147.8, 144.3, 143.7, 141.0, 140.5, 140.3, 128.0, 127.7, 124.0, 31.2; MS EI (m/z) $357\left(\mathrm{M}^{+}, 36 \%\right), 284(15), 283$ (7), $236(8), 194$ (14), 193 (89), 192 (25), 179 (6), 165 (100); Anal: calculated for $\mathrm{C}_{14} \mathrm{H}_{11} \mathrm{~N}_{7} \mathrm{O}_{3} \mathrm{~S}(357.349) \mathrm{C}, 47.06 ; \mathrm{H}, 3.10 ; \mathrm{N}, 27.44$, Found: C, 47.06; H, 3.09; N, 27.46\%.

\section{N'-[(E)-(3-Nitrophenyl)Methylidene]-2-(9H-Purin-6-ylsulfany} l)acetohydrazide (4)

$N^{\prime}$-[(E)-(3-nitrophenyl)methylidene]-2-(7H-purin-6ylsulfanyl)acetohydrazide was prepared from $2(0.226 \mathrm{~g}, 1$ $\mathrm{mmol})$ and 3-nitrobenzaldehyde $(0.166 \mathrm{~g}, 1.1 \mathrm{mmol})$ in ethanol $(20 \mathrm{~mL})$ with a few drops of acetic acid.

Pale yellow solid: mp $260{ }^{\circ} \mathrm{C}$, Yield $79 \%,{ }^{1} \mathrm{H}$ NMR $\delta(400$ MHz, DMSO-d $\left.\mathrm{d}_{6}\right) 13.53(1 \mathrm{H}, \mathrm{s}), 12.01(1 \mathrm{H}, \mathrm{s}), 11.84(1 \mathrm{H}, \mathrm{s})$, $8.68(1 \mathrm{H}, \mathrm{s}), 8.66(1 \mathrm{H}, \mathrm{s}), 8.51-7.70(4 \mathrm{H}, \mathrm{m}), 4.70(2 \mathrm{H}, \mathrm{s})$; ${ }^{13} \mathrm{C}$ NMR $\delta$ (100 MHz, DMSO-d 6 ) 169.2, 154.4, 153.2, 144.4, 141.1, 133.2, 132.9, 130.4, 124.2, 124.1, 121.1, 121.0, 31.3; MS EI (m/z) $357\left(\mathrm{M}^{+}, 23 \%\right), 284$ (25), 195 (6), 194 (10), 193 (100); Anal: calculated for $\mathrm{C}_{14} \mathrm{H}_{11} \mathrm{~N}_{7} \mathrm{O}_{3} \mathrm{~S}$ (357.349) $\mathrm{C}, 47.06$; H, 3.10; N, 27.44\% , Found: C, 47.05; H, 3.10; N, 27.45\%.

\section{$N^{\prime}-[(E)-(2-N i t r o p h e n y l) m e t h y l i d e n e]-2-(9 H-P u r i n-6-$}

ylsulfanyl)acetohydrazide (5)

$N^{\prime}$-[(E)-(2-nitrophenyl)methylidene]-2-(7H-purin-6ylsulfanyl) acetohydrazide was prepared from $2(0.226 \mathrm{~g}, 1 \mathrm{mmol})$ and 2-nitrobenzaldehyde $(0.166 \mathrm{~g}, 1.1 \mathrm{mmol})$ in ethanol $(20 \mathrm{~mL})$ with a few drops of acetic acid.

Light yellow solid; mp $248{ }^{\circ} \mathrm{C}$; Yield $85 \% ;{ }^{1} \mathrm{H}$ NMR $\delta$ (400 MHz, DMSO-d $\left.{ }_{6}\right) 13.54(1 \mathrm{H}, \mathrm{s}), 12.11(1 \mathrm{H}, \mathrm{s}), 8.95(1 \mathrm{H}$, s), $8.76(1 \mathrm{H}, \mathrm{s}), 8.65-7.64(5 \mathrm{H}, \mathrm{m}), 4.25(2 \mathrm{H}, \mathrm{s}) ;{ }^{13} \mathrm{C} \mathrm{NMR} \delta$ (100 MHz, DMSO-d $\left.{ }_{6}\right)$ 169.3, 154.2, 153.3, 151.3, 148.9, 143.7, 133.6, 130.6, 130.5, 128.5, 128.0, 128.0, 124.6, 31.1; MS EI $(\mathrm{m} / \mathrm{z}) 357\left(\mathrm{M}^{+}, 15 \%\right) 284$ (28), 209 (5), 194 (12), 193 (100); Anal: calculated for $\mathrm{C}_{14} \mathrm{H}_{11} \mathrm{~N}_{7} \mathrm{O}_{3} \mathrm{~S}(357.349) \mathrm{C}, 47.06 ; \mathrm{H}, 3.10$; N, 27.44\%, Found; C, 47.03; H, 3.06; N, 27.36\%.

\section{$N^{\prime}$-[(E)-(4-Chlorophenyl)methylidene]-2-(6,9-Dihydro-5H-} Purin-6-ylsulfanyl)acetohydrazide (6)

$N^{\prime}$-[(E)-(4-chlorophenyl)methylidene]-2-(6,7-dihydro-5H-purin-6-ylsulfanyl)acetohydrazide was prepared from $2(0.226 \mathrm{~g}$,
$1 \mathrm{mmol})$ and 4-chlorobenzaldehyde $(0.154 \mathrm{~g}, 1.1 \mathrm{mmol})$ in ethanol $(20 \mathrm{~mL})$ with a few drops of acetic acid. The product was filtered, washed and recrystallized with ethanol.

Pale yellow solid; mp $210{ }^{\circ} \mathrm{C}$; Yield $68 \%$; ${ }^{1} \mathrm{H}$ NMR $\delta$ (400 MHz, DMSO-d $\left.)_{6}\right) 13.52(1 \mathrm{H}, \mathrm{s}), 13.49(1 \mathrm{H}, \mathrm{s}), 11.84(1 \mathrm{H}$, s), $11.66(1 \mathrm{H}, \mathrm{s}), 8.70(1 \mathrm{H}, \mathrm{s}), 8.40-7.48(4 \mathrm{H}, \mathrm{m}), 4.63(2 \mathrm{H}$, s); ${ }^{13} \mathrm{C}$ NMR $\delta\left(100 \mathrm{MHz}\right.$, DMSO-d $\left.{ }_{6}\right) 169.5,151.3,151.2$, 142.0, 136.0, 133.0, 132.6, 130.0, 129.0, 128.8, 128.7, 128.4, 30.1; MS EI (m/z) $346\left(\mathrm{M}^{+}, 51 \%\right), 280$ (6) 279 (9), 278(34), 277 (27), 276 (52) 167 (36), 165 (100); Anal: calculated for $\mathrm{C}_{14} \mathrm{H}_{11} \mathrm{ClN}_{6} \mathrm{OS}(346.796) \mathrm{C}, 48.21 ; \mathrm{H}, 3.76 ; \mathrm{N}, 24.09 \%$, Found; C, $48.19 ; \mathrm{H}, 3.72 ; \mathrm{N}, 24.10 \%$.

2-(6,9-dihydro-5H-purin-6-ylsulfanyl)-N'-[(E)-phenylmethylid ene]acetohydrazide (7)

2-(6,7-dihydro-5H-purin-6-ylsulfanyl)- $N^{\prime}-[(E)$-phenylmethylid ene]acetohydrazide was prepared from $2(0.226 \mathrm{~g}, 1 \mathrm{mmol})$ and benzaldehyde $(0.116 \mathrm{~g}, 1.1 \mathrm{mmol})$ in ethanol $(20 \mathrm{~mL})$.

Yellow solid: mp $266{ }^{\circ} \mathrm{C}$; Yield $66 \%$; ${ }^{1} \mathrm{H}$ NMR $\delta(400$ $\left.\mathrm{MHz}, \mathrm{DMSO}-\mathrm{d}_{6}\right) 12.68(1 \mathrm{H}, \mathrm{s}), 11.91(1 \mathrm{H}, \mathrm{s}), 8.67(1 \mathrm{H}, \mathrm{s})$, $8.47(1 \mathrm{H}, \mathrm{s}), 8.33(1 \mathrm{H}, \mathrm{s}), 8.25-7.90(5 \mathrm{H}, \mathrm{m}), 4.26(2 \mathrm{H}, \mathrm{s})$; ${ }^{13} \mathrm{C}$ NMR $\delta$ (100 MHz, DMSO-d $)$ 169.4, 153.4, 151.3, 146.8, 143.3, 131.3, 130.0, 129.8, 128.9, 128.3, 127.0, 126.9, 126.8, 31.2; MS EI (m/z) 313( $\left.\mathrm{M}^{+}, 26 \%\right), 281$ (6), 280 (19), 239 (6), 238 (8), 208 (13), 207 (13), 194 (17), 193 (100); Anal: calculated for $\mathrm{C}_{14} \mathrm{H}_{12} \mathrm{~N}_{6} \mathrm{OS}(312.351) \mathrm{C}, 53.49 ; \mathrm{H}, 4.49 ; \mathrm{N}, 26.73 \%$, Found; C, 53.48; H, 4.41; N, 26.71\%.

2-(6,9-Dihydro-5H-purin-6-ylsulfanyl)-N'-[(E)-(2-methoxyphe nyl)methylidene]acetohydrazide (8)

2-(6,7-dihydro-5H-purin-6-ylsulfanyl)- $N$ '-[(methoxyphenyl)m ethylidene]acetohydrazide was prepared from $2(0.226 \mathrm{~g}, 1$ $\mathrm{mmol})$ and 3-methoxybenzaldehyde $(0.149 \mathrm{~g}, 1.1 \mathrm{mmol})$ in ethanol $(20 \mathrm{~mL})$.

Yellow solid: mp $180{ }^{\circ} \mathrm{C}$; Yield $61 \%$; ${ }^{1} \mathrm{H}$ NMR $\delta(400$ MHz, DMSO-d $\left.{ }_{6}\right) 13.54(1 \mathrm{H}, \mathrm{s}), 11.76(1 \mathrm{H}, \mathrm{s}), 8.92(1 \mathrm{H}, \mathrm{s})$, $8.67(1 \mathrm{H}, \mathrm{s}), 8.65(1 \mathrm{H}, \mathrm{s}), 8.36-7.16(4 \mathrm{H}, \mathrm{m}), 4.22(2 \mathrm{H}, \mathrm{s})$, $3.91(3 \mathrm{H}, \mathrm{s}) ;{ }^{13} \mathrm{C}$ NMR $\delta\left(100 \mathrm{MHz}, \mathrm{DMSO}-\mathrm{d}_{6}\right)$ 168.9, 158.7 , 157.6, 156.5, 151.3, 142.9, 142.3, 131.5, 131.3, 126.5, 122.1, 120.7, 111.8, 55.8, 31.1; MS EI (m/z) $342\left(\mathrm{M}^{+}, 26 \%\right), 310(6)$, 268 (7), 237(20), 195 (6), 194 (10), 193 (100); Anal: calculated for $\mathrm{C}_{15} \mathrm{H}_{14} \mathrm{~N}_{6} \mathrm{O}_{2} \mathrm{~S}$ (342.377) C, 52.31; H, 4.68; N, 24.40\%, Found;C, 52.33; H, 4.67; N, 24.41\%.

2-(6,9 -Dihydro-5H-Purin-6-ylsulfanyl)-N'-[(E)-(2-hydroxyph enyl)methylidene]acetohydrazide (9)

2-(6,7-dihydro-5H-purin-6-ylsulfanyl)- $N^{\prime}$-[(E)-(2-hydroxyphe nyl)methylidene]acetohydrazide was prepared from $2(0.226$ $\mathrm{g}, 1 \mathrm{mmol})$ and salicyaldehyde $(0.134 \mathrm{~g}, 1.1 \mathrm{mmol})$ in ethanol $(20 \mathrm{~mL})$.

Brown needles; mp $224{ }^{\circ} \mathrm{C} ;{ }^{1} \mathrm{H}$ NMR $\delta(400 \mathrm{MHz}$, DMSO-d $\left._{6}\right) 12.98(1 \mathrm{H}, \mathrm{s}), 11.13(1 \mathrm{H}, \mathrm{s}), 8.99(1 \mathrm{H}, \mathrm{s}) 8.42$ $(1 \mathrm{H}, \mathrm{s}), 8.36(1 \mathrm{H}, \mathrm{s}), 8.17(1 \mathrm{H}, \mathrm{s}), 7.69-7.94(5 \mathrm{H}, \mathrm{m}), 3.33$ $(2 \mathrm{H}, \mathrm{s}) ;{ }^{13} \mathrm{C}$ NMR $\delta\left(100 \mathrm{MHz}, \mathrm{DMSO}-\mathrm{d}_{6}\right)$ 169.4, 153.4, $158.5,145.9,144.4,133.2,130.7,129.7,128.8,128.7,127.2$, 126.8, 126.8, 32.0; MS EI $(\mathrm{m} / \mathrm{z}) 328\left(\mathrm{M}^{+}, 15 \%\right), 297$ (5), 
296 (13), 268 (12), 254 (14), 240 (68), 223 (40), 194 (15), 193 (100); Anal: calculated for $\mathrm{C}_{14} \mathrm{H}_{12} \mathrm{~N}_{6} \mathrm{O}_{2} \mathrm{~S}$ (328.350) C, $50.90 ; \mathrm{H}, 4.27 ; \mathrm{N}, 25.44 \%$, Found; C, 50.91; H, 4.26; N, $25.43 \%$.

$N^{\prime}-\{(1 Z)-[4-(D i m e t h y l a m i n o) p h e n y l] M e t h y l i d e n e\}-2-(9 H-$ purin-6-ylsulfanyl)acetohydrazide (10)

$N^{\prime}$-\{(1Z)-[4-(Dimethylamino)phenyl]methylidene $\}-2-(9 H$-purin-6-ylsulfanyl)acetohydrazide was prepared from $2(0.226$ $\mathrm{g}, 1 \mathrm{mmol})$ and 4-(dimethylamino)benzaldehyde $(0.152 \mathrm{~g}, 1.1$ $\mathrm{mmol})$ in ethanol $(20 \mathrm{~mL})$.

Yellow solid; mp $259{ }^{\circ} \mathrm{C}$; Yield $76 \% ;{ }^{1} \mathrm{H}$ NMR $\delta(300$ MHz, DMSO-d $\left.{ }_{6}\right)$ 13.52 $(1 \mathrm{H}, \mathrm{s}), 11.54(1 \mathrm{H}, \mathrm{s}), 8.69(1 \mathrm{H}, \mathrm{s})$, $8.49(1 \mathrm{H}, \mathrm{s}), 8.09-7.47(5 \mathrm{H}, \mathrm{m}), 4.15(2 \mathrm{H}, \mathrm{s}), 3.18(6 \mathrm{H}, \mathrm{s})$; ${ }^{13} \mathrm{C}$ NMR $\delta_{\mathrm{C}}(75 \mathrm{MHz}$, DMSO-d 6 ) 169.4, 151.3, 151.3, 147.0, 145.3, 144.2, 142.9, 141.7, 129.4, 128.3, 128.2, 128.0, 127.6, 111.7, 44.3, 31.1; MS EI (m/z) $355\left(\mathrm{M}^{+}, 28 \%\right), 323(14), 294$ (17), 239 (6), 251 (16), 208 (15), 193 (100); Anal: calculated for $\mathrm{C}_{16} \mathrm{H}_{17} \mathrm{~N}_{7} \mathrm{OS}(355.419) \mathrm{C}, 54.07 ; \mathrm{H}, 4.82 ; \mathrm{N}, 27.59 \%$, Found; C, 54.07; H, 4.81; N, 27.58\%.

$N^{\prime}-\{(1 Z)-[4-(D i e t h y l a m i n o)$ phenyl]Methylidene $\}-2-(9 H-p u r i n-$ 6-ylsulfanyl)acetohydrazide (11).

$N^{\prime}$-\{(1Z)-[4-(Diethylamino)phenyl]methylidene $\}$-2-(9H-purin6-ylsulfanyl)acetohydrazide was synthesized from $2(0.226 \mathrm{~g}, 1$ $\mathrm{mmol}$ ) and 4-(diethylamino)benzaldehyde $(0.190 \mathrm{~g}, 1.1 \mathrm{mmol})$ in ethanol $(20 \mathrm{~mL})$.

Yellow solid: mp $246{ }^{\circ} \mathrm{C}$; Yield $78 \%$; ${ }^{1} \mathrm{H}$ NMR $\delta(300$ MHz, DMSO-d $\left.\mathrm{d}_{6}\right) 13.36(1 \mathrm{H}, \mathrm{s}), 1.69(1 \mathrm{H}, \mathrm{s}), 8.44(1 \mathrm{H}, \mathrm{s})$, $8.23(1 \mathrm{H}, \mathrm{s}), 8.12-7.57(5 \mathrm{H}, \mathrm{m}), 4.21(2 \mathrm{H}, \mathrm{s}), 3.62(4 \mathrm{H}$, quart, $\mathrm{J}=7.20), 1.08(6 \mathrm{H}, \mathrm{t}, \mathrm{J}=7.20) ;{ }^{13} \mathrm{C} \mathrm{NMR} \delta(75 \mathrm{MHz}$, DMSO-d $_{6}$ ) 168.3, 151.6, 151.3, 149.2, 145.3, 44.3, 142.8, $141.6,129.7,128.2,128.3,128.9,127.7,110.9,43.7,32.8$, 12.4; MS EI (m/z) $383\left(\mathrm{M}^{+}, 17 \%\right), 350$ (57), 349 (10), 335 (21), 307 (8), 291 (14), 263 (13), 175 (11), 160 (30), 147 (17), 133 (100); Anal: calculated for $\mathrm{C}_{18} \mathrm{H}_{21} \mathrm{~N}_{7} \mathrm{OS}$ (383.47) C, 56.38; H, 5.52; N, 25.57\%, Found;C, 56.39; H, 5.51; N, $25.56 \%$.

\section{Assessment of antithyroid activity}

In vitro

The formation of charge transfer (CT) complex between the compound and iodine is an in vitro indication of antithyroid effects of any compound. The complexation of the compounds with iodine was studied spectrophotometrically. The compounds were found to be soluble only in DMSO, therefore, fresh solutions of the compounds and iodine were prepared in DMSO and added simultaneously in the reaction cell in 1:1 volumetric ratio. Iodine has absorption maximum in the visible region, while the compounds exhibited $\lambda_{\max }$ in UV region [11]. Therefore, as a strategy the solutions of iodine and the compounds as were used in the reaction cell, were kept in reference cell in visible and UV regions, respectively, to nullify the absorbance due to free iodine and the compounds in the respective regions. The iodine concentration was fixed at $10^{-5} \mathrm{M}$, while that of compounds was varied between $10^{-4}$ and $10^{-3} \mathrm{M}$. The spectra were recorded immediately on double beam UV-visible spectrophotometer (UVD-3500 Labomed Inc.). The formation constants were calculated on the basis of spectrophotometric data using Lang's method [12].

In vivo

Male Wistar rats of 4 weeks age and $150 \pm 25 \mathrm{~g}$ weight were obtained from National Institute of Health, Islamabad (Pakistan) and were acclimatized with laboratory environment for 7 days before start of experiment at Department of Zoology University of the Punjab Lahore (Pakistan). The temperature and relative humidity were kept at $25 \pm 1,55 \pm 10 \%$ with 12 hour light/dark cycle. They were fed with chick feed with water ad libitum.

Before the administering chemicals, the animals were assigned different groups (comprising 5 animals each) in such a way that mean body weight of all the groups was almost the same. Fresh solutions of the compounds (including MMI) were prepared in vehicle (DMSO) and were injected via i.p. injection at a dose rate of $20 \mathrm{mg} / \mathrm{kg}$ daily in the morning for 15 days. The control and vehicle control group received equivalent dose of normal saline and DMSO, respectively. Standard laboratory procedures were adopted for housing and experimentation on rats [13].

Daily cage-side observations were performed to detect moribund or dead rats. On the morning of $16^{\text {th }}$ day the rats were weighed and shifted to dissection room and were kept there quietly for an hour prior to blood sampling to avoid hormonal variations due to transfer stress. The blood samples were collected by puncturing abdominal aorta under light diethyl ether anesthesia. Serum was separated by centrifuging the blood samples at $4000 \mathrm{rpm}$ and was stored at $-40{ }^{\circ} \mathrm{C}$ until the hormonal analysis. The free $\mathrm{T}_{3}$ and $\mathrm{T}_{4}$ concentrations were measured with commercially available RIA kits of Immunotech France, while TSH levels was determined with ELISA rat TSH kit purchased from Cusabio Biotech Co. Limited. After blood sampling the animals were dissected under deep diethyl ether anesthesia. Thyroid was dissected out from one animal from each group animal and placed in Petri dish containing saline solution to clear organ from fats and blood. The tissues were then weighed with the help of Sartorius digital balance and were fixed in 4\% PFA for 4-6 hours. Thyroid body indices (TBIs), which may be defined as weight of clean thyroid tissues $(\mathrm{mg})$ before fixation per $100 \mathrm{~g}$ of final body weight, were calculated for every animal. Slides of $5 \mu \mathrm{m}$ thin sections of thyroid were prepared and stained with hematoxylin and eosin stains. Olympus BX51microscope fitted with Olympus DP12 digital camera was used to study the histopathology of the tissues, which were photographed at $20 \mu \mathrm{m}$ scale.

The hormonal data was analyzed using Student's "t" test, which is a useful hypothesis testing method for small sample sizes $(<30)$. The "t" values (with respect to vehicle control) determined from the assay results were compared with those of the table values at $5 \%$ and $1 \%$ probability $(d . f .=8$ and $\alpha=$ $0.05,0.01)$ [14]. 


\section{Acknowledgements}

The authors acknowledge the support of the Higher Education Commission of Pakistan for financial help. The services of the Department of Zoology, University of the Punjab Lahore and Centre for Nuclear Medicine, Mayo Hospital, Lahore are also acknowledged by the authors.

\section{References}

1. Cooper, D. S. Lancet 2003, 62, 459-468.

2. Reid, J. R.; Wheeler, S. F. Am. Fam. Physician. 2005, 72, 623630.

3. Bandyopadhyay, U.; Biswas, K.; Banerjee, R. K. Toxicol. Lett. 2002, 128, 117-127.

4. Abou-Shaaban, R. A.; Al-Khamees, H. A.; Abou-Auda, H. S.; Simonelli, A. P. Saudi Pharm. J. 1995, 3, 156 - 175.
5. Raby, C.; Lagorce, J. F.; Jambut-Absil, A. C.; Buxeraud, J.; Catanzano, G. Endocrinology 1990, 126, 1683-1691.

6. Pullman, B.; Pullman, A. Biochemistry 1958, 44, 1197-1202.

7. (a) Fatima, I.; Munawar, M. A.; Khan, M. A.; Tasneem, A.; Khalil, M. J. Braz. Chem. Soc. 2010, 21, 1699-1703; (b) Fatima, I.; Munawar, M. A.; Tasneem, A. J. Mex. Chem. Soc. 2010, 54,227232.

8. Fatima, I.; Munawar, M. A.; Tasneem A.; Jahan, S.; Khan M. A. J. Serb. Chem. Soc. 2011, 76, 1355-1364.

9. Durgun, B.; Capan, G.; Ergenç, N.; Rollas, S. Pharmazie 1993, 48, 942-943

10. Lagorce, J. F.; Comby, F.; Rousseau, A.; Buxeraud, J.; Raby, C. Chem. Pharm. Bull. 1993, 41, 1258-1260.

11. Barett, A. J.; Barett, K. L. Khan, A. J. Macromol. Sci. A 1998 , 35,1603-1611.

12. Lang, R. P. J. Am. Chem. Soc. 1962, 84, 1185-1192.

13. The Council of European Communities, Council Directive 86/609/ EEC (1986).

14. Saroj, K.P. Statistics for Geoscientists-Techniques and Applications, Concept Publishing Co., New Delhi, India, 1997, p. 288. 